\title{
DETERMINATION OF THE DEGREE OF UNIFORMITY OF BARS FOR MAGNETIC STANDARDS
}

\author{
By Raymond L. Sanford, Assistant Physicist
}

\section{STATEMENT OF PROBLEM}

Concrete magnetic standards, consisting of straight bars of magnetic material selected and prepared with a view to obtaining the maximum degree of permanence and homogeneity, are of very great value in magnetic testing. By means of readings taken on such standardized bars, permeameters are calibrated and various methods of magnetic testing are compared with a standard method. One of the most important requirements of a magnetic standard bar is that it shall be magnetically uniform along its length. All precision methods for the magnetic measurement of straight bars assume uniformity along the length of the specimen. If this condition is not met, errors may arise, due to the effect of poles which modify the distribution of magnetomotive force. These errors may be of considerable magnitude and can not be calculated or eliminated from the measurements.

Burrows, ${ }^{1}$ in his paper on the Determination of the Magnetic Induction in Straight Bars, calls attention to the necessity for uniformity and shows the effect of nonuniformities on the distribution of magnetic induction in a bar magnetized in a doubleyoke apparatus. He points out the fact that numbers or identification marks should not be punched or stamped on those portions of a bar which are to be included in the magnetic measurements. Several methods have been suggested ${ }^{2}$ for testing the uniformity

\footnotetext{
${ }^{1}$ Burrows, this Bulletin, 6, p. 59; 1909 .

2 Ebeling and Schmidt, Wied. Ann. 58, p. 330, 1896, examine different portions of long bars by measurements with a single-yoke apparatus.

Ebeling, Wied. Ann. 58, p. 342, r896, examines portions of the bars mentioned above by determining the electrical resistivity of different portions of their length.

Kann Phys. Zs., 7, p. 526, 1906, suggests the detection of flaws by a determination of the mutual induc tance between two windings on a core which is arranged to include the material under test.

McCann and Colson, Western Electrician, 43, p. 76, 1908, investigate the continuity of wire ropes, such as mine hoists, etc., by observing changes in self-inductance of a coil surrounding the wire rupe under test by means of an alternating current and suitable instruments.

Pierce, Proc. Am. Acad. of Arts and Sciences, 46, p. x97, 1910, determined the suitability of cast-iron pieces for use as permanent magnets by a resistance method.
} 
of bars and the detection of flaws. No one, however, has indicated the magnitude of the effect of nonuniformities on the accuracy of thagnetic measurements.

This paper (I) describes a method for determining the degree of magnetic uniformity along the length of a straight bar, (2) indicates the magnitude of the effect of nonuniformities on the accuracy of magnetic measurements, and (3) gives a criterion for the degree of uniformity of magnetic standards.

\section{THEORY OF THE METHOD}

Uniformity measurements, as carried out in the present investigation, are made by observing the distribution of magnetic leakage along the length of a specimen when it is magnetized between the poles of a suitable electromagnet. If the bar is uniform, the magnetic induction and leakage vary along its length in a regular manner. Nonuniformities are indicated by irregularities in the distribution of induction and leakage along the bar. In this work the leakage is defined as the change of induction per unit length along the bar. In other words, the leakage at any point on the bar is the first derivative of the induction with respect to the displacement. The curve expressing the rate of change of leakage along the bar, or the leakage slope, has been chosen to represent the degree of magnetic uniformity and is referred to hereafter as the "uniformity curve." This curve gives the second derivative of the induction with respect to the displacement.

The curves given in Fig. I show the distribution of induction and leakage for two bars, one of which is uniform while the other has a magnetically hard spot near one end. The induction is greatest at the ends and has a minimum value near the middle of the bar. If the bar is uniform, the induction varies in such a way that the leakage curve is a straight line having a slope which depends upon the magnetic hardness of the material. If the magnetic circuit is symmetrical and the two joint contacts have the same magnetic reluctance, the leakage has a zero value at the middle of the bar. If the two joints are not of the same reluctance, the zero point may be displaced, but the slope remains the same. Consequently, the "uniformity curve" for a uniform bar is a straight line parallel to the axis of the bar. If a bar is nonuniform, the leakage does not vary at a constant rate and the nature and position of the nonuniformity is shown by the deviation of the uniformity curve from a straight line. An increase in the 
value of the ordinate at any point along the length of the bar indicates a magnetically hard spot, while a decrease in its value shows a soft spot.
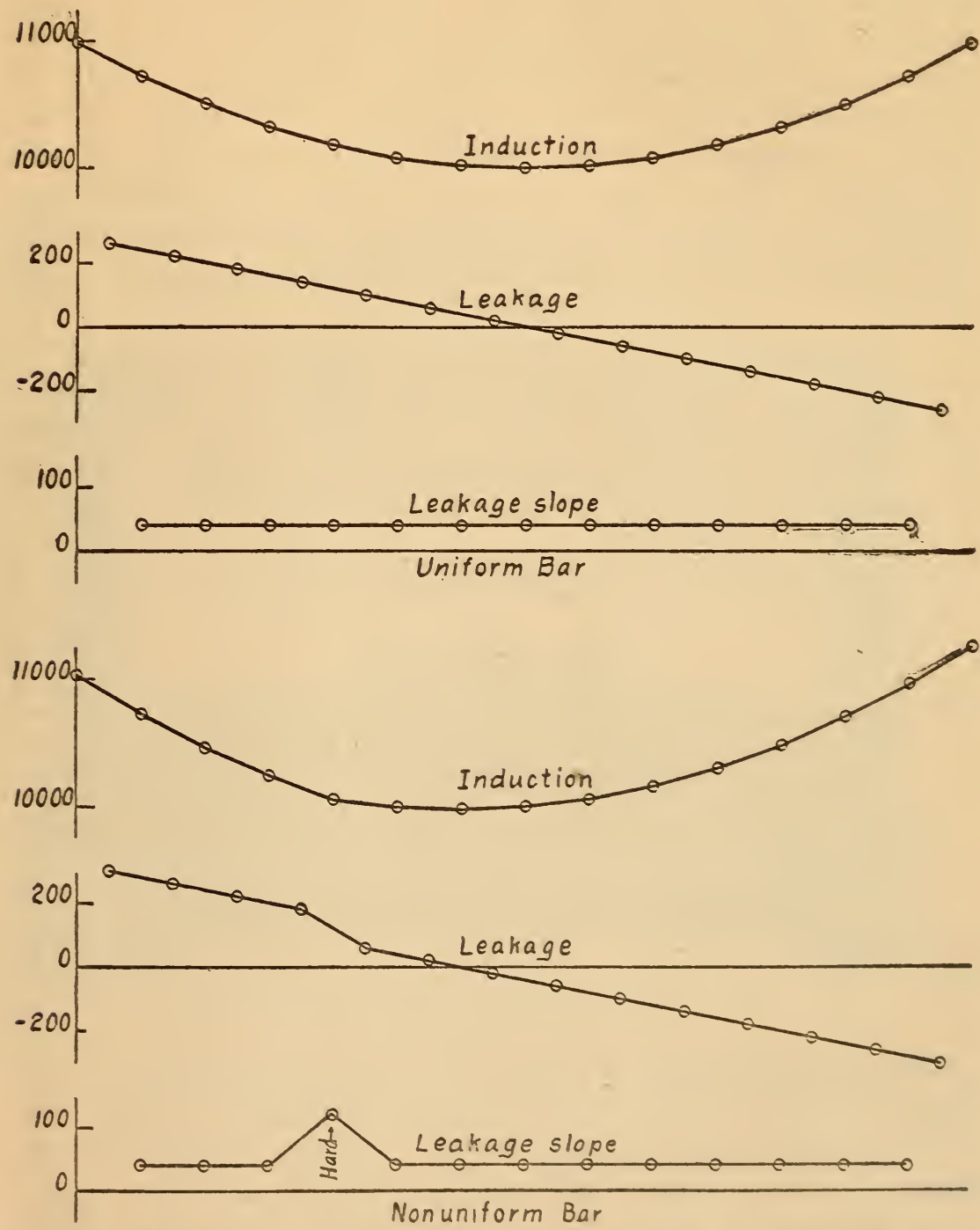

FIG. I.-Distribution of magnetic induction and leakage for bars magnetizut between the poles of an electromaynet

\section{DETAILS OF THE METHOD}

The essential features of the apparatus used in this investigation consist of an electromagnet and a pair of specially wound test coils (Fig. 2). The electromagnet has pole pieces adapted for 
clamping the specimens. The two test coils, which are slipped over the bar under test, are of Ioo turns each and are wound on a common form. Each coil extends over a space of $\mathrm{I} \mathrm{cm}$ and the distance between centers is $2 \mathrm{~cm}$. The displacement of the coils along the bar is read by means of a suitable scale mounted on the apparatus. The ballistic galvanometer is calibrated by means of a mutual inductance, and its sensitivity and damping are adjusted by means of the usual combination of series and parallel resistances. With a single test coil in circuit, the galvanometer deflection on reversal of the magnetizing current is proportional to the induction in that part of the bar under the test coil. With

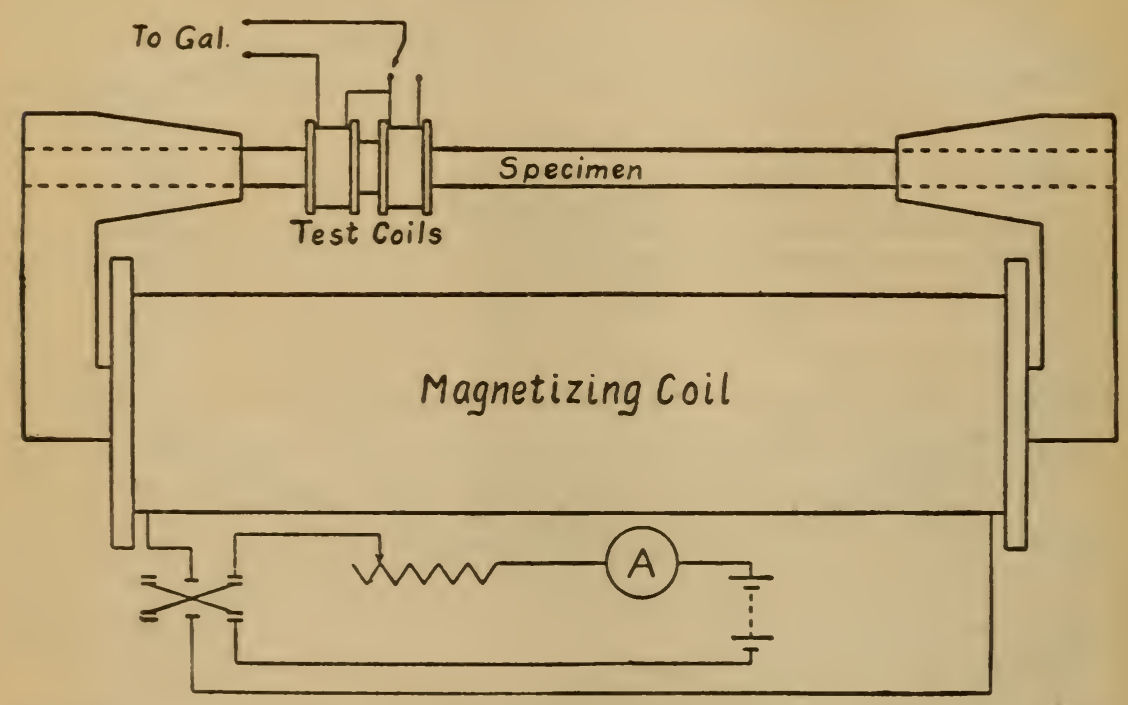

FIG. 2.-Diagram of apparatus for the determination of magnetic uniformity along the length of a straight bar

the two coils connected in opposition, the deflection on reversal is proportional to the difference in induction between the parts of the bar under the two coils. This difference divided by the distance between coils is the average leakage over that region. From leakage data taken at equal intervals along the length of the bar the ordinates of the uniformity curve are calculated, and these points are plotted against displacement along the bar.

A continuous record for the whole length of the bar is possible by a modification of the apparatus. If the opposed test coils are moved at a uniform rate along the bar, the emf induced is proportional to the rate of change of leakage along the bar. This 
Bulletin Bureau of Standards, Vol. 14

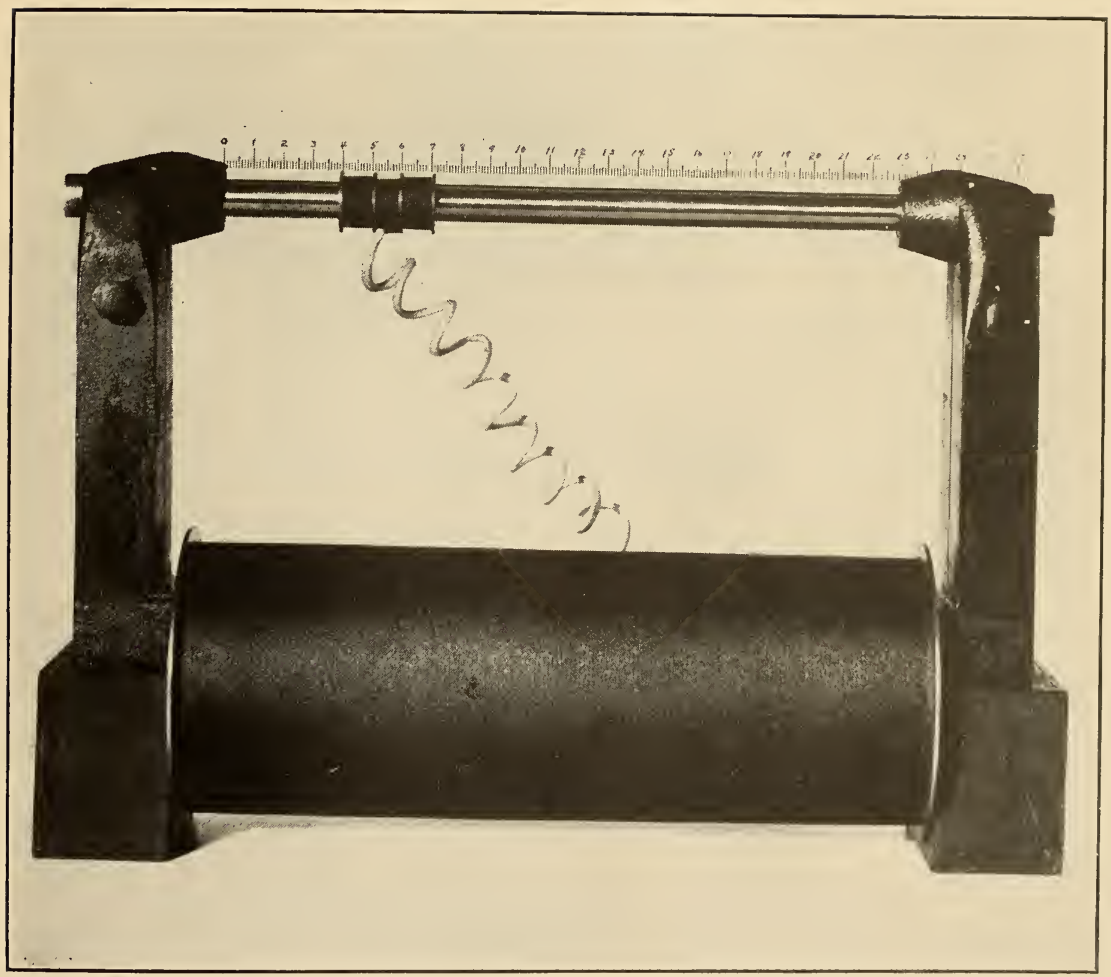

FIG. 3.-Apparatus for the determination of magnetic uniformity along the length of a straight bar 
emf is constant for a uniform bar and variations indicate nonuniformities. It is possible with suitable apparatus to obtain a photographic record showing the degree of uniformity along the length of a bar.

The choice of the induction at which to make uniformity determinations is of considerable importance. If the induction is carried too high, the effect is to raise the value of the ordinates of the

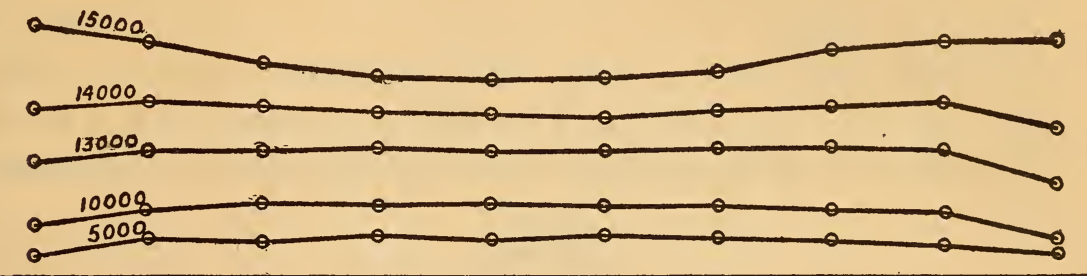

FIG. 4.-Form of the uniformity curve taken at different values of induction

uniformity curve near the ends (Fig. 4). This effect is probably due to the high magnetizing force necessary to produce high inductions. Readings taken at low inductions may be affected by the previous magnetic history of the specimen. The best region in which to work is that in which the induction is changing at the greatest rate, but this involves a preliminary determination of the normal induction, which is not desirable. All of the curves in this paper were taken with an induction at the middle of the bar of approximately ro ooo gausses. This induction for ordinary

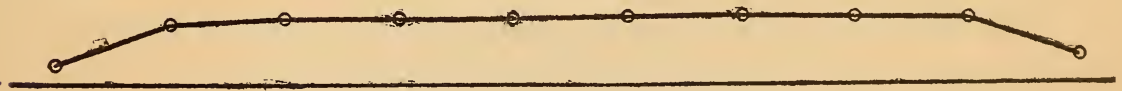

FIG. 5.-Showing depression of the ends of the uniformity curve for a uniform bar due to the effect of the poles of the electromagnet

materials does not require an excessive magnetizing force and gives the required sensitivity.

The form of the uniformity curve is affected by the poles of the electromagnet which modify the distribution of induction near the ends of the bar. The effect is to depress the curve at the ends (Fig. 5). This limits the proportion of the total length of the bar that can be examined by this method. This limitation is not serious, however, as the ends of the bar are usually clamped in yokes or pole pieces and do not enter directly into the measurements. Values can be obtained for points near the ends of the 
bar by the use of extensions of the same cross section, which are butted against the ends of the bar and held in place by means of iron sleeves. This arrangement allows readings to be taken nearer the ends of the bar, as the effect of the poles is decreased and the effect of the additional joints is of less importance.

The consistency on repetition is satisfactory, as shown by repeated tests on the same bars. The results can be duplicated within the limits of the precision of reading the galvanometer and setting the position of the test coils. The two curves of Fig. 6 were taken nearly a year apart. The slight differences are immaterial and may be attributed to different positions of the bar in the apparatus and consequent different settings of the test coils.
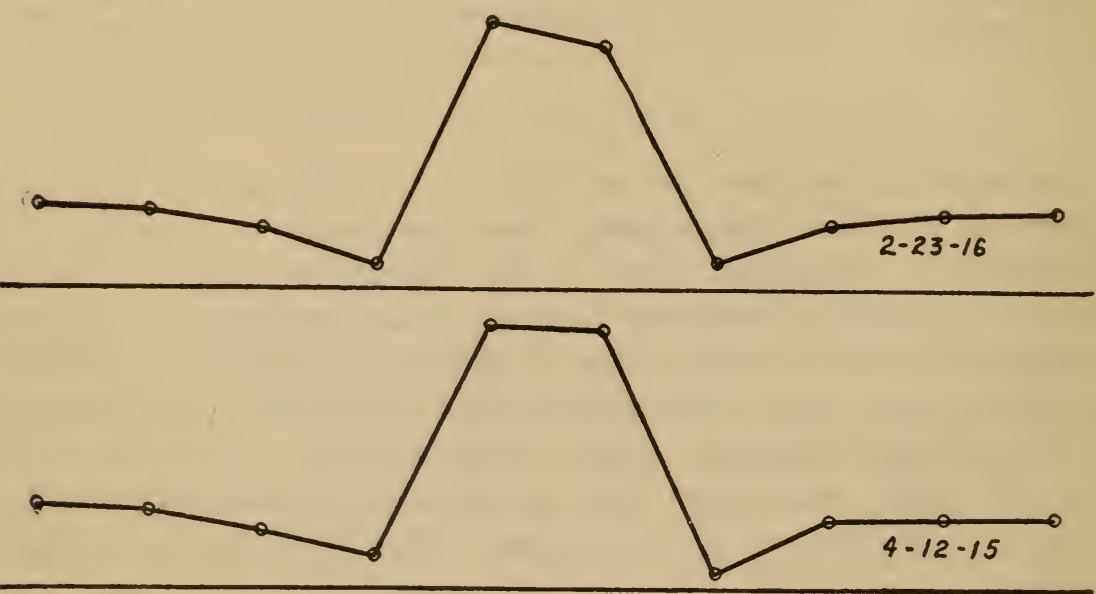

FIG. 6.-Showing the consistency on repetition of the uniformity curve

\section{OBSERVATIONS AND RESULTS}

Tests have been made on a large number of bars to determine the degree of magnetic uniformity that may be expected in ordinary test bars as received and also to determine the magnitude of the effect of nonuniformities on the accuracy with which magnetic measurements can be made.

The curves of Fig. 7 give the results of uniformity tests on a number of cast-steel bars that have been submitted to the Bureau of Standards for test. The curves all show large deviations from uniformity and are characteristic of this class of material. Most of these bars have smooth surfaces and give no indication to the eye of the presence of nonuniformities. Another class of material that gives trouble is wrought iron, which in general is 
very inhomogeneous. Uniformity curves (Fig. 8) were taken for I 2 bars of this material, which had been carefully annealed. These curves also show large deviations and indicate extremely inhomogeneous material.

The effect of nonuniformities on the accuracy of the magnetic measurements may be illustrated by the results of tests on a long bar which has a magnetically soft spot near the middle. Normal induction curves (Fig. 9) taken with the middle test coil of a Burrows permeameter over different portions of this bar show that the observed values of the magnetizing force necessary to produce given inductions are lower when the test coil is over the region which is magnetically soft as indicated by the uniformity curve. In this case there are differences as great as 15 per cent in the observed values of the magnetizing force giving the same induction.

A quantitative statement of the effect of nonuniformities on the accuracy of magnetic measurements is difficult because the magnitude of the error depends upon a number of variables. Among these variables are the induction in the specimen, the nature, number, magnitude, position, and extent of the nonuniformities present and the type of apparatus used for the measurements. It is possible, however, to set a limit for the magnitude of nonuniformity that may exist, when a certain accuracy is desired, from the effect on the normal induction measurements of a single nonuniformity which is located in the position of maximum effect. The effect on the data obtained with the Burrows permeameter is greatest when the nonuniformity is at the middle of the bar. For the purpose of setting this limit, normal induction measurements were made with a Burrows permeameter on a number of bars of various materials which originally were approximately uniform. After nonuniformities had been artificially produced at the middle, the measurements of uniformity and normal induction were repeated. The curves of Fig. Io which show the effect of drilling a $2.5 \mathrm{~mm}$ hole in a bar of low carbon steel $1.27 \mathrm{~cm}$ in diameter are typical. The effect of these artificially produced hard spots is to increase the observed value of the magnetizing force necessary to produce a given induction. The data show that for any given induction the percentage error in the observed magnetizing force is, roughly, proportional to the percentage deviation of the ordinate of the uniformity curve from the mean value. Observations made with a number of modifications of the 

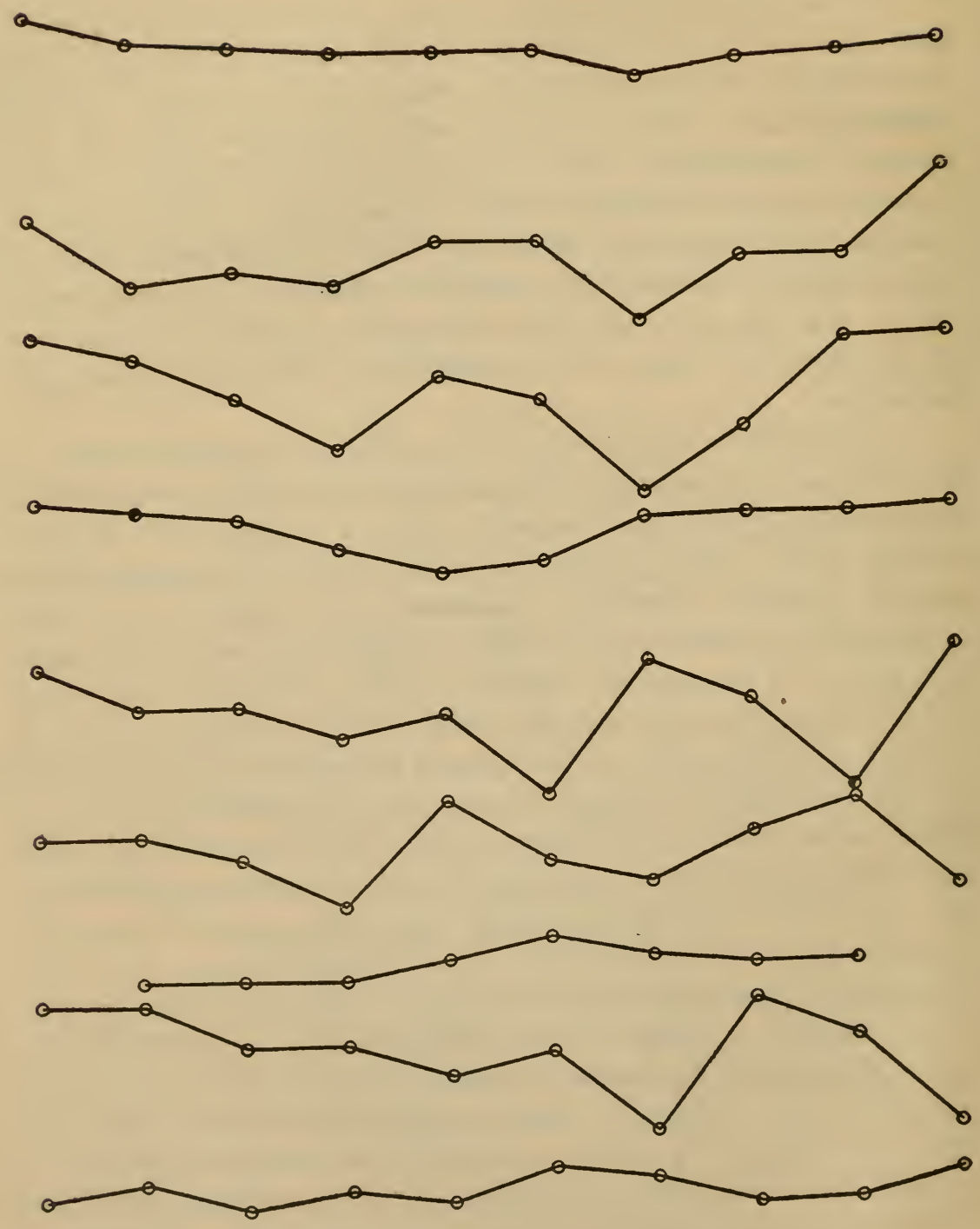

FIG. 7.-Uniformity curves for a number of characteristic cast-iron bars. 

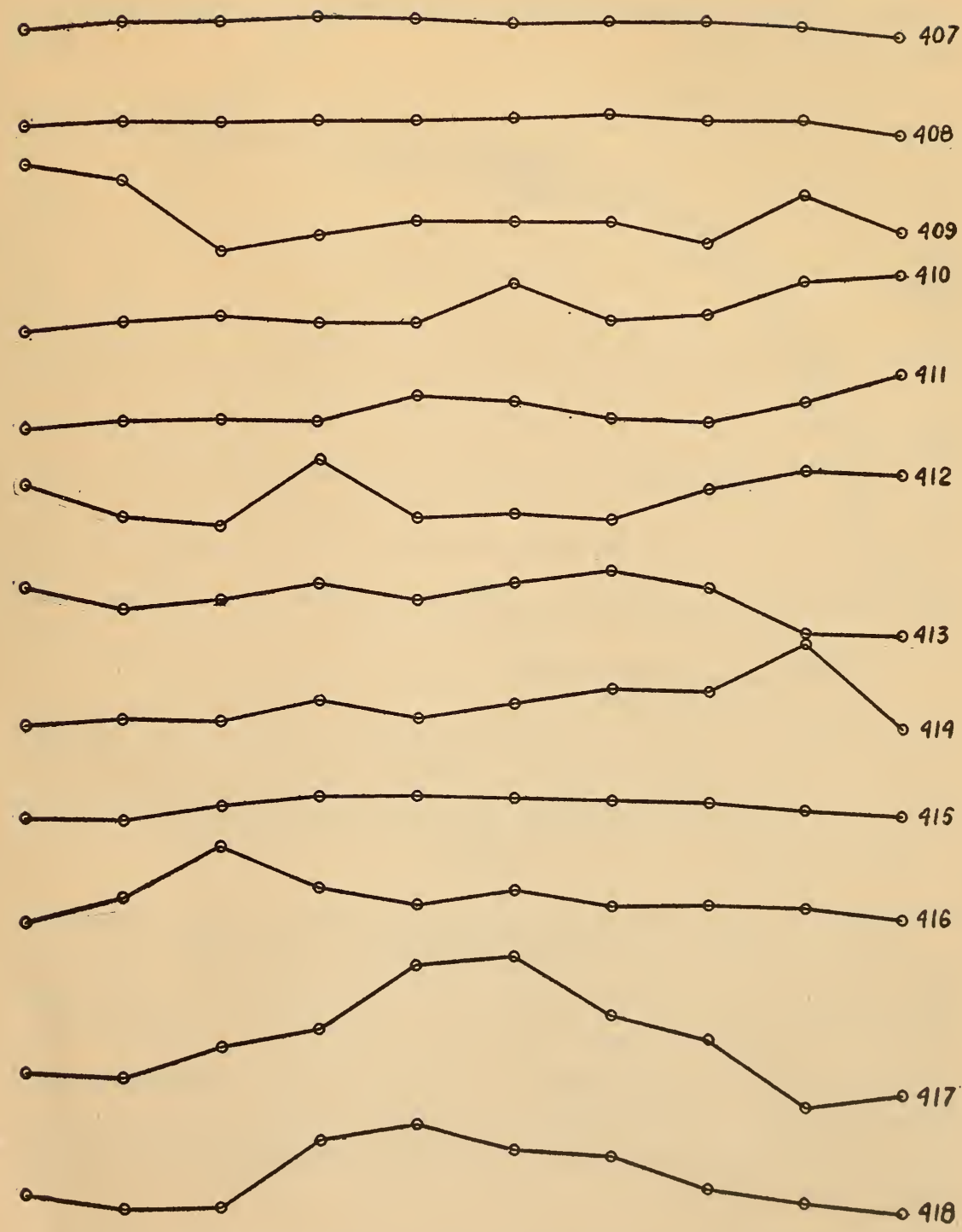

FIG. 8.-Uniformity curves for twelve specially prepared bars of zorought iron 
form of the magnetizing system give substantially the same results. The quantitative results may, however, be affected by variations in the proportions of the apparatus. With the apparatus used it was found that for an accuracy of I per cent variations in the value of the ordinate of the uniformity curve from the mean must not exceed ro per cent.

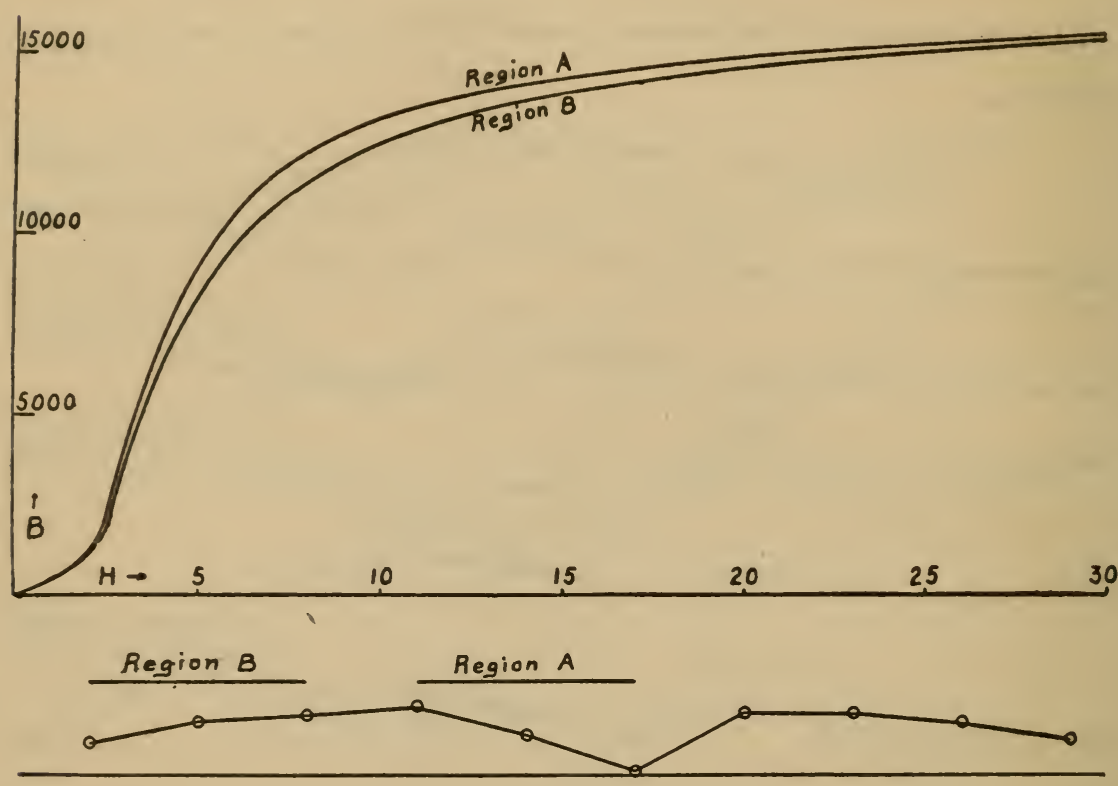

Fig. 9.-Normal induction curves taken with the permeameter test coils over the regions $A$ and $B$ as indicated on the uniformity curve, showing the effect of the magnetically soft spot near region $A$

Numerical data for the 12 wrought-iron bars of Fig. 7 (Table I) show that not one of these bars comes within this limit. The best one shows a maximum deviation of 16 per cent and may be used where an accuracy better than 2 per cent is not necessary. The next best bar has a maximum deviation of 38 per cent and is used as an auxiliary. If two bars have equal maximum deviations, the one whose deviations have the smaller arithmetic sum is the better. 

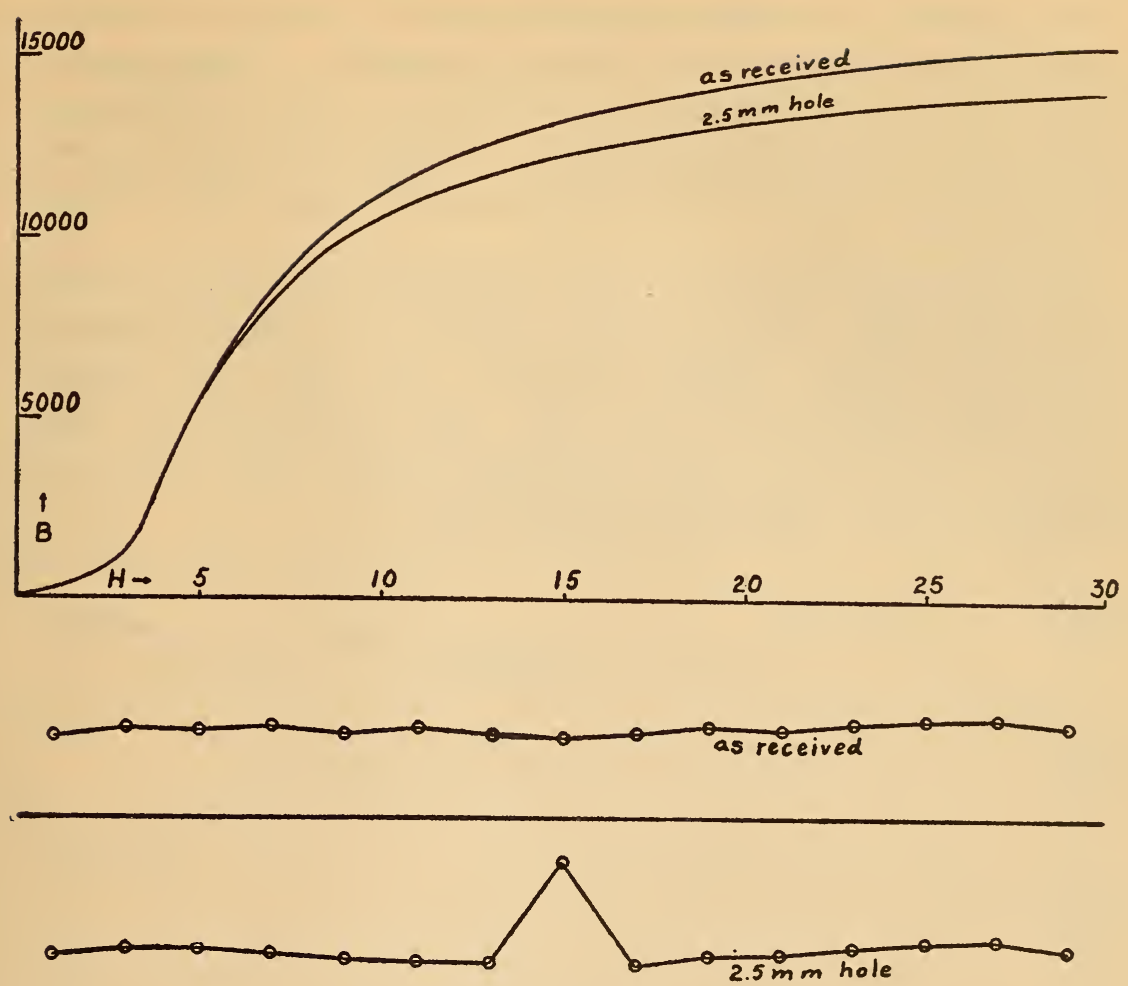

FrG. I0.-Showing the effect on uniformity and normal induction curves of drilling a $2.5 \mathrm{~mm}$ hole through the middle of a bar $1.25 \mathrm{~cm}$ in diameter

\section{TABLE 1}

Per Cent Deviation From the Mean of Ordinates of the Uniformity Curves for 12 Wrought-Iron Bars

\begin{tabular}{|c|c|c|c|c|c|c|c|c|c|c|c|c|}
\hline Bar No... & 407 & 408 & 409 & 410 & 411 & 412 & 413 & 414 & 415 & 416 & 417 & 418 \\
\hline stance from middle: & & & & & & & & & & & & \\
\hline $6 \mathrm{~cm} .$. & -2 & -15 & +288 & -37 & -48 & -48 & -56 & -78 & -104 & -22 & -100 & -103 \\
\hline $4 \mathrm{~cm} . . . . . .$. & -2 & -15 & -250 & +4 & -30 & -103 & -22 & -96 & -13 & +203 & -45 & -98 \\
\hline $2 \mathrm{~cm} .$. & +15 & +4 & -100 & -46 & -48 & +232 & +24 & +39 & +54 & +19 & -17 & +73 \\
\hline $0 \mathrm{~cm} \ldots$ & +8 & -8 & +31 & -50 & +122 & -58 & -26 & -78 & +46 & -58 & +93 & +110 \\
\hline $2 \mathrm{~cm} . .$. & -16 & +12 & +25 & +208 & +83 & -35 & +24 & -17 & +25 & -3 & +108 & +42 \\
\hline $4 \mathrm{~cm} . .$. & +1 & +38 & +25 & -25 & -35 & -68 & +58 & +113 & +13 & -72 & +2 & +25 \\
\hline $6 \mathrm{~cm} \ldots \ldots . .$. & -2 & -8 & -200 & +12 & -57 & +84 & +4 & +83 & -17 & -69 & -43 & -64 \\
\hline
\end{tabular}


The magnetic laboratory of the Bureau of Standards maintains a set of magnetic standards of different materials. A large number of bars have been examined and only those whose uniformity is satisfactory are included in this set. (The wrought-iron bar mentioned above is included for want of a better bar of this class of material.)

The uniformity curves for these standard bars, a few of which are given in Fig. II, show that the comparative magnetic hardness is indicated by the average ordinate. The higher the ordinate the harder magnetically is the bar. The numerical data (Table 2) show that, with the single exception already noted, the deviations are all well within the specified limit.

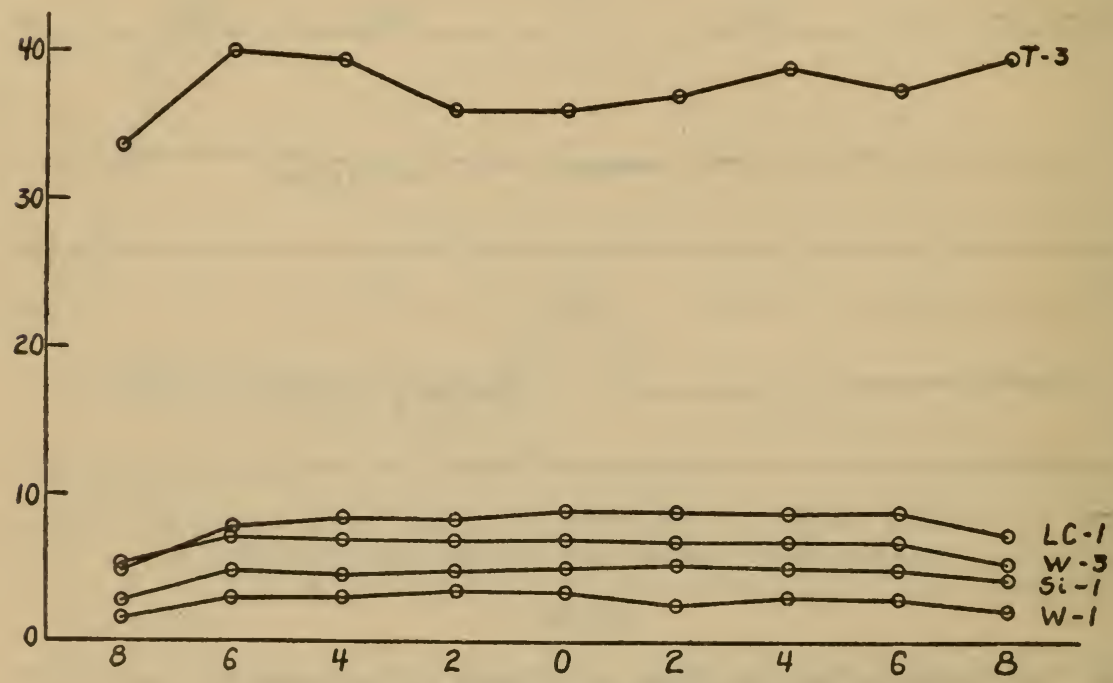

FIG. II.-Uniformity curves for a number of magnetic standard bars.

TABLE 2

Per Cent Deviation From the Mean of Ordinates of the Uniformity Curves for Five Standard Bars

\begin{tabular}{|c|c|c|c|c|c|}
\hline Bar No............. & W-1 & W-3 & Si-1 & LC-1 & $\mathbf{T}-3$ \\
\hline \multicolumn{6}{|l|}{ Distance from middle: } \\
\hline $6 \mathrm{~cm} \ldots \ldots \ldots \ldots$ & -2 & +3 & -3 & -8 & +6 \\
\hline $4 \mathrm{~cm} \ldots \ldots \ldots$ & -2 & +2 & -7 & -1 & +5 \\
\hline $2 \mathrm{~cm} \ldots \ldots \ldots \ldots \ldots \ldots \ldots \ldots \ldots \ldots$ & +15 & 0 & -1 & -2 & -5 \\
\hline $0 \mathrm{~cm} \ldots .$. & +8 & +2 & +3 & +5 & -5 \\
\hline (n................. & -16 & -3 & +5 & +5 & -2 \\
\hline$\ldots \ldots \ldots \ldots \ldots$ & +1 & 0 & +3 & 0 & +3 \\
\hline $6 \mathrm{~cm} \ldots .$. & -2 & -2 & +1 & +2 & -1 \\
\hline
\end{tabular}




\section{OTHER APPLICATIONS}

The application of this method to the detection of mechanical inhomogeneities and flaws opens up a wide field of possible applications. Space will only be taken here to describe the results of one out of a number of such tests that have already been made.

Fig. 12 gives uniformity curves for a bar which was originally uniform. After the bar had been bent in a vise till it had a permanent bend of about $10^{\circ}$ and then straightened the uniformity curve shows a decided kink which indicates a magnetically hard spot. The effect is shown to be greatest at the point where the bar was clamped and gradually decreases toward the free end of the bar. After annealing to remove the effect of these strains, the uniformity curve shows even less deviation than the original.

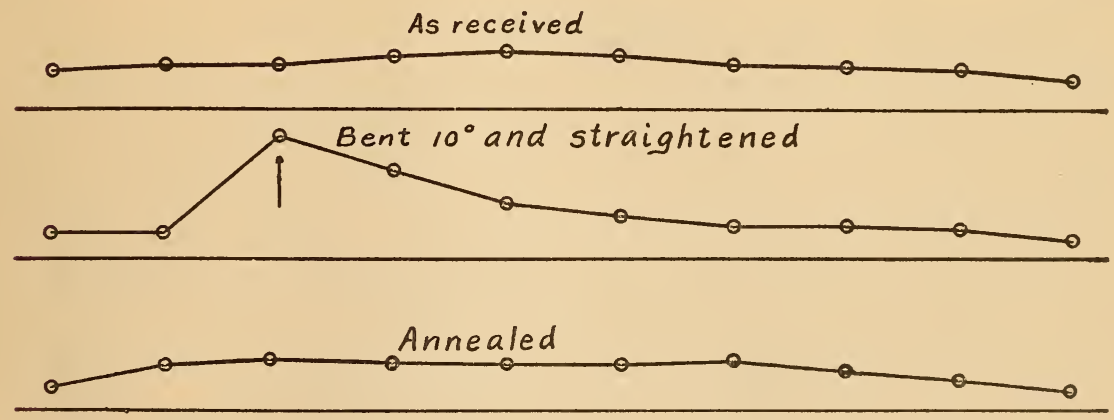

FIG. I2.-Showing the effect of bending and restraightening and of annealing on the magnetic uniformity along the length of a bar.

\section{SUMMARY}

Magnetic standard bars are used for the calibration of permeameters and the comparison of methods of magnetic testing with a standard method. One requisite of a magnetic standard bar is that it shall be magnetically uniform along its length. If this condition is not met, errors may arise which can not be calculated or eliminated from the measurements, and which may be of considerable magnitude.

The degree of magnetic uniformity of a bar may be determined from observations of the distribution of magnetic leakage along the length of a specimen when it is magnetized between the poles of a suitable electromagnet. The degree of uniformity is indicated by values of the rate of change of leakage along the length of the bar. Deviations of these values from a constant indicate the presence of nonuniformities. An increase in the value indicates a 
magnetically hard spot, while a decrease indicates a soft spot. The degree of uniformity can be indicated by means of a curve plotted between values of the rate of change of leakage and displacement along the bar. This curve is called the uniformity curve and for a uniform bar is a straight line parallel to the axis of displacement.

Errors in magnetic measurements due to nonuniformities depend upon the induction, the nature, location, magnitude, and extent of the nonuniformities and upon the type of apparatus. For a given accuracy no ordinate of the uniformity curve must deviate from the mean by more than a given amount.

This method may be applied to the examination of magnetic materials for mechanical inhomogeneities and the detection of flaws.

WASHINGTON, July 26, r9i6. 